\title{
Exploring the Power Clean
}

Thomas Huyghe ${ }^{1,2}$, Brent Goriss 33 , Ernest DeLosAngeles ${ }^{4,5}$, Stephen P. Bird 5 ,6

${ }^{1}$ UCAM Research Center for High Performance Sport, Catholic University San Antonio, Murcia, Spain, ${ }^{2}$ International Sports Management and Business Department, Sports Studies, Amsterdam University of Applied Sciences,

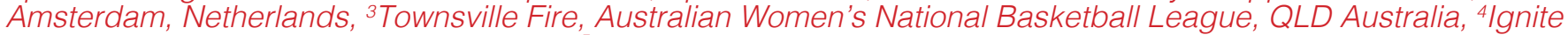
NBA G League, California, United States, ${ }^{5}$ Sport and Exercise Science Research Group, Centre for Health Research, School of Health and Wellbeing, University of Southern Queensland, QLD Australia, ${ }^{6}$ Basketball New Zealand, Wellington New Zealand

\begin{abstract}
The power clean and its variations are prescribed by strength and conditioning coaches as part of the "big three' to develop "total body strength". This article explores the application of the power clean and its variations to athletic performance and introduces strength and conditioning coaches to teaching progressions, with specific emphasis on developing the correct body positioning required for the power clean. Teaching components are addressed with special reference to taller athletes. It is recommended that strength and conditioning coaches teaching the hang clean follow a progression model to decrease movement complexity when advancing athletes to the power clean.
\end{abstract}

\section{POWER CLEAN TERMINOLOGY}

When prescribing weightlifting derivatives to enhance athletic performance, strength and conditioning coaches often think of the power clean (PC), with teaching instructions dating back to the 1980's [6, 14, 20, 27]. However, the term 'clean' refers to a large collection of weightlifting-type pulling exercises [9, 37, 38], with these movement patterns designed to enhance explosive strength linked to athletic performance [19]. The clean movement can be performed with a number of variations which primarily relate to the starting position (Table 1) and may be performed from a static position off technique blocks or with the bar lowered to a hang position at the knee, for example from the high, mid and low pull position $[8,33]$. The 'clean pull' variation involves the phase between the first and second pull of the movement only [9], whereas the power clean, hang power clean and mid-thigh power clean all involve the first pull, transition, second pull, catch and recovery phase $[8,11,12]$. The terminology used to describe power clean variations are consistent throughout the literature, with variations in the starting position of the bar in the hang clean with the bar starting from either the thighs $[11,12]$ or knees [8].

\section{RESEARCH EXAMINING THE POWER CLEAN}

To date, the majority of research examining the power clean and its variations has focussed on two aspects, these being 1) kinetic and kinematic outcomes; and 2) teaching progression models. Firstly, the kinetic outcomes achieved by performing power clean derivatives, based on their starting positions (i.e., off technique blocks or from the high, mid or low pull position) result in observed differences in kinetic and kinematic patterns between novice and skilled lifters. Kipp et al. [23] examined the kinetic and kinematic patterns of the hip and knee joints when performing a power clean (85\% 1RM) to identify associations between weightlifting biomechanics and performance. A greater lift mass was associated with less hip extension motion during the first pull and second-knee bend transition, a smaller knee extension moment during the first pull, and a greater a knee extension moment during the second pull. Additionally, faster and earlier temporal transition from knee flexion to extension at the beginning of the second pull was also associated with higher lift mass. Notably, the two kinematic patterns correlated with weightlifting performance were related to hip motion characteristics: 1) The correlation between hip joint 
extension motion during the first pull and rapid extension during the second pull, relative 1RM, links steady and controlled hip motion to greater relative lift mass; and 2) A smaller hip joint motion during the transition between the first pull and second pull is significantly correlated to greater relative $1 \mathrm{RM}$. This suggests the importance of rapid hip and trunk motion along with knee extension moments in relation to weightlifting performance.

A valuable coaching reference suggested by Stone and colleagues [29] is that of torso angle remaining constant and controlled during the first pull, which is associated with higher load lifted. Anecdotally, our experience with taller athletes (i.e., basketball players) suggests they are more likely to exhibit forward trunk flexion and instability of the trunk during the initial phase leading into the first pull. This in turn will result in the lifter performing the first pull with the lower back as the shoulders do not remain vertically aligned over the mid-foot. Such positioning may unfavourably increase hip joint motion leading into the transition phase and second pull, resulting in larger hip joint motion between the transition and second pull. This reduces the athlete's ability to rapidly triple extend in the second pull, as hip extension and knee extension are required to be performed over a greater range of motion. Stone et al. [29] suggest that such reference points will assist coaches to effectively teach power clean techniques, especially to taller athlete, with significant improvements in bar path reported within 4-weeks of coaching.

Winchester and colleagues [40] report that during the transition between the first pull and second pull, the highest rate of force development and peak force expression occurs, which highlights the importance of both a slight increase in knee joint motion and rapid transition from knee flexion to knee extension at the beginning of the second pull. In the transition phase, taller lifters tend to exhibit less knee flexion during the double knee bend, accompanied by forward trunk flexion. Comparatively, greater peak extension motions of the hip and knee are reported for highly skilled world class lifters compared with skilled collegiate lifters during the first and second pull phases [5]. Elite weightlifters extend their knee and ankle joints more rapidly during these phases [15]. Strength and conditioning coaches should pay particular attention to the applied force onto the barbell from the first pull with different kinematic patterns of knee and hip flexion and extension, and hip and knee joint motion of taller lifters, as this will affect the kinetic output of the lift. For example, excessive hip flexion during the transition phase is detrimental as too much hip flexion-extension motions may lead to excessive "hipping" of the barbell and cause undesirable barbell trajectories associated with unsuccessful weightlifting attempts. A more common outcome when observing taller athletes.

From a coaching perspective, biomechanical determinants are a significant contributing factor to the success of the hang power clean, especially for taller athletes at higher loads [5, 23, 39]. Given the complexity of the such weightlifting movements, kinetic and kinematic analysis, although not easily quantified, is a valuable tool for strength and conditioning coaches to provide instruction focusing on proper bar path during the movement [39]. Strength and conditioning coaches are encouraged to use visual and verbal feedback to track bar path with athletes learning power clean and its variations, with the kinematics of the lift represented by trunk, hip and knee patterns of movement. There are many software programs on the market that may be used to assist the strength and conditioning coach such as Spark Motion Pro, Coach's Eye and Form Check.

Secondly, in order to reinforce proper technique, several teaching progressions models have been proposed for the power clean to assist athletes achieving technical proficiency. Hedrick [17] outlines a 12-step progression model for teaching the power clean, which utilises a sequential order of performance. It is suggested that due to the complex nature of teaching the clean, each step in the teaching progression should build on the previous technical proficiency, therefore, making such complex skill acquisition easier. However, given the complexity of the power clean, Duba and colleagues [11] highlight the importance of teaching and mastery of the hang power clean preceding the teaching of the power clean. The authors outlined an initial 6-step teaching progression model, however this may be reduced to a 4-step teaching model when progressing from the hang power clean to the power clean [12]. Two exercises considered essential in the execution of the hang power clean are the Romanian deadlift $(\mathrm{RDL})$ and the front squat (FSq) [3, 4]. Specifically, the postural positioning and flexibility required for successful execution of the RDL and FSq greatly influences an athlete's technical proficiency in the hang power clean. The recommended 6-step hang power clean teaching progression [11], and subsequent 4-step teaching model [12] progressing to the power clean are based upon the different phases of the exercise. With emphasis on execution 
Table 1. Overview of power clean variations and sport-specific applications

Power Clean Variation

Primary Muscles Used

\section{Comments}

The Olympic lift that involves pulling from the

floor and catching in a squat is multi-joint uti-

lizing a fast movement velocity. Often used

Clean
$\mathrm{BB} / \mathrm{KB} / \mathrm{DB} / \mathrm{MB} / \mathrm{LM}$

Gluteus, quadriceps, spinal erectors, abdominals, quadratus lumborum

by athletes to enhance muscular power and strength.

The hang starting position can be beneficial for those with mobility restrictions. Used to increase explosive performance of the lower

\section{$\mathrm{BB} / \mathrm{KB} / \mathrm{DB} / \mathrm{MB} / \mathrm{LM}$}

\section{Jump Shrug}

$\mathrm{BB} / \mathrm{TB} / \mathrm{KB} / \mathrm{DB} / \mathrm{MB} / \mathrm{LM}$

\section{Clean Pull}

$\mathrm{BB} / \mathrm{TB} / \mathrm{KB} / \mathrm{DB} / \mathrm{MB} / \mathrm{LM}$

\section{Muscle Clean}

\section{Power Clean}

\section{Sandbag Clean}

\section{Water Bag Clean}

\section{KB Clean}

DA/SA

\section{DB Clean}

DA/SA
Gluteus, hamstrings, spinal erectors, abdominals, quadratus lumborum.

Gluteus, hamstrings, spinal erectors, abdominals, quadratus lumborum, calves.

Hip adductors/abductors, spinal erectors, abdominals.

Shoulder musculature, upper back quadriceps.

Gluteus, hamstrings, spinal erectors, uppe back, spinal erectors.

Anterior deltoid, External oblique, Erector spinae, Gluteus medius.

Anterior deltoid, External oblique, Erector spinae, Gluteus medius.

Anterior deltoid, Quadriceps, Hamstrings, Gluteus maximus.

Anterior deltoid, Quadriceps, Hamstrings, Gluteus maximus. body.

$$
\text { body. }
$$

Loads greater than 1RM of clean can be applied. Can also be implemented during speed-strength phase with light-moderate loads.

Easy to teach and execute for novice lifters.

It is versatile at various speeds and start

heights. Can be performed at loads greater than 1RM of clean.

Helpful to learn and reinforce proper upper body mechanics of the clean leading into front rack position.

This variation is caught in a quarter squat position. Enhances explosive power of the lower body.

Allows for variations in movement degrees and beneficial for building work capacity.

Involves greater core muscle activation and a reduced load placed on lower back.

Efficient way of teaching the clean to novice

lifters. Is an effective complementary method to plyometrics and other techniques to enhance strength and power.

The ability to train the movement unilaterally Beneficial for movement restrictions or in rehabilitation.
Sport-Specific Applications

Weightlifting, Football, Rugby, Track and Field, Wrestling.

Football, Rugby, Track and Field, Wrestling.

Basketball, Volleyball, Soccer, Baseball.

Basketball, Volleyball, Baseball, Soccer.

Weightlifting, Basketball, Volleyball.

Weightlifting, Football, Basketball, Baseball, Volleyball.

Early off season.

Late phase rehabilitation.

Football, Baseball, Basketball, Volleyball, Tennis, Wrestling.

Football, Baseball, Basketball, Volleyball, Tennis, Wrestling 
mastery of the hang power clean, the first pull and transition phase of the power clean are integrated into the second pull and catch phase of the hang power clean (Figure 1).

\section{APPLICATION TO SPORT}

From an athletic development perspective, both the acute (short-term) and chronic (long-term) application of the power clean and its weightlifting variations have been linked to athletic preparation programming. For instance, many athletes seek enhanced speed strength capabilities with power development the primary physiological characteristic determining successful athletic performance [18]. As power output is one of the most important factors in the athletic performance [1, 2, 16], there is much interest in the transfer-of-training effect of weightlifting exercises such as the hang power clean and power clean, and potential effectiveness to improve the athlete's capability of power, and subsequently athletic performance. Importantly, from an athletic perspective, Hori and colleagues [19] examined whether athletes with high performance in a hang power clean transfers to high performances in sprinting, jumping and change of direction. The authors reported that performance of $1 \mathrm{RM}$ hang power clean could differentiate performance of jumping and sprinting. Athletes in the top half of
1RM hang power clean (relative to body mass) had higher performance of jumping and sprinting, demonstrating higher maximum strength (1RM front squat both absolute and relative), and higher peak power output (counter-movement jump; CMJ 40kg relative; and CMJ relative). That is to say - athletes with high performance in the 1RM hang power clean possesses greater maximum strength and power deemed essential for peak performance of jumping and sprinting. Relative 1RM hang power clean, front squat, power output in CMJ 40 and CMJ, jump height, and time in the 20-m sprint were significantly correlated, ranging from $r=0.51-0.60$. This is of potential significance to strength and conditioning coaches as it is reasonable to assume that the hang power clean shares similar strength qualities required for fundamental athletic performance tasks such as jumping and sprinting.

Examining the differences in peak vertical ground reaction force $\left(F_{z}\right)$ and rate of force development (RFD) in elite rugby league players, Comfort et al. [8] had athletes perform one set of three repetitions at $60 \%$ one-repetition maximum (1RM) (power clean) of the power clean, hang power clean, mid-thigh power clean and mid-thigh clean pull. The mid-thigh variations produce significantly greater $F_{z}$ compared to the power clean and hang power clean when performed at the same relative load (60\% 1RM). It was hypothesized that the higher $F_{z}$ outputs between

\section{Power clean}

- Teach as one movement

- Practice 3-5 reps with lighter loads

- Control speed of $1^{\text {st }}$ pull and transition

\section{Clean deadlift + hang power clean \\ - Combo of two pre-requisites for power clean \\ Brief 1-2 s pause between transition \& $2^{\text {nd }}$ pull \\ - Progression to one smooth movement}

\section{Clean deadlift}

- Teach starting position, $1^{\text {st }}$ pull \& transition

- Emphsize power \& strength stance, \& posture position

- Progression to first pull and transition phase

\section{Develop correct hang power clean technique}

Foundation for successful power clean progression

Figure 1. Four-step teaching progression for the power clean. The clean deadift and clean deadlift + hang power clean combo provide the foundation for successful exercise progression. Adapted from Duba et al. [12]. 
the mid-thigh variations was due to their kinematic similarity, and the reduced displacement when performing the technique. The authors suggested that greater loads (>100\% 1RM power clean) can be used when performing the mid-thigh clean pull and would therefore be best implemented in a maximal strength phase, whilst the mid-thigh hang power clean would be used in a power phase due to its ability to maximize $F_{z}$ and RFD.

From a coaching point of view, the mid-thigh power clean and mid-thigh clean pull have practical benefits for less experienced athletes as they are easier to learn and require less technical experience [8]. The lower limb kinematics during the mid-thigh variations have been reported to replicate the joint angles achieved during phases of running and jumping. Research indicates that hang power clean peak power occurs at submaximal loads (70\% 1RM power clean) [21], however, power output will progressively decrease with the performance of multiple continuous repetitions. Therefore, training in a cluster set configuration with rest periods of 10 to 30 seconds between repetitions may help athletes maximise and maintain power output compared to traditional protocols where repetitions are done continuously without rest [35]. From a sport-specific point of view, we have successfully used such an approach with professional basketball players. Additionally, within the professional basketball environment, we have witnessed the preferred type of power clean variations used amongst elite basketball players to be the hang power clean and mid-thigh clean pull. As previously reported, from a the kinetic outcomes perspective, $F_{z}$ and RFD have been shown to be maximised in a hang power clean and mid-thigh clean pull [8], which are highly desirable for anaerobically-based sport athletes, where sprinting, jumping, and dynamically change of direction are critical elements. Research by Hori et al. [19] demonstrated that higher performance of the 1RM hang power clean was associated with higher performance in such elements.

The kinematic demands on range of motion and joint angle during the performance of the hang power clean and mid-thigh clean pull are less demanding on athletes with longer limbs compared to the traditional power clean from the floor. Athletes with longer limbs are required to undergo greater ranges of motion, especially in the lower body, during the first pulling phase of a power clean from the floor. The correct starting position from the floor is often challenging for longer-limbed athletes to attain. As such, the first pull is performed through a greater joint angle range of motion and does not produce the maximal drive force to transfer into the transition phase and second pull, thereby limiting the RFD. The mid-thigh clean pull has also been successfully used amongst taller athletes demonstrating limited ability to perform the catch phase of the hang power clean effectively. The catch phase can be challenging due to the slow rotation of the elbows underneath the bar with loads of more than $60-70 \% 1 \mathrm{RM}$ power clean. The athletes have also found it challenging to catch the bar with the elbows elevated and facing forwards, rather catching with the elbows facing downwards and depressed. This ultimately places more load bearing stress on the wrists, which is a common complaint amongst taller athletes. To alleviate this issue, given the athlete's training history and technical ability, we have coached two modifications to the lift. One is the addition of a potentiated pull immediately prior to commencing the hang clean. The athlete performs one repetition of a potentiated pull, briefly resets and immediately performance the hang clean. The second modification is the addition of a no catch release, thereby alleviating eccentric loading and force absorption of the catch. Verbal cues include (i) Drive everything from the floor as one; (ii) Shoulders to your ears; and (iii) Pull under the bar.

\section{Teaching Components}

The following brief overview provides explanation for the teaching components of the hang clean:

1. Setup: The hang power clean begins from the hang position, which is the position at which the 'second pull' (the most powerful fragment of the movement) in the power clean exercise commences (Figure 2) [11, 32, 33]. In preparation of performing the hang clean, technique boxes (lifting blocks) or safety bars of a squat rack should be oriented in front of the patella region of the athlete (relative to the athlete's anthropometrics), above the proximal attachment of the patellar tendon [33]. Once the setup is completed, the athlete stands with his or her feet approximately shoulder width apart and holds the bar in "hook grip" (fingers over thumb). Following correct hand and feet placement, the athlete bends the knees, pushing the hips back as they descend into a hang position with the bar located right above knee level (quarter to half front squat), with the eyes kept up and forward. The bar is vertically aligned with the midfoot keeping toes slightly pointed outward. Strength and conditioning coaches may use various action and posture cues to get into a safe and effective 
hang position such as "back tight", "get tall", "push the hips back", "lean over the bar", "keep the bar close", "long arms", "elbows out", "sit on the heels" as common examples based upon individual perceptions and adaptations [11]. In turn, this will enable the athlete to avoid 'energy leaks'throughout the movement, improve control over the bar, and consequently produce the greatest possible forces into the ground while assuring proper and safe movement quality.

2. Execution: In order to proceed from the initial hang position towards the peak power position, a "tight" torso must be maintained ensuring muscular tension in the hamstrings, glutes, and lower back (erector spinae muscles) facilitated by a deep inhale prior to the ascent [11, 33]. When the athlete transitions towards the peak power position, his or her back extends and hips move forward at the same instant while the bar ascends vertically (up and into the body). To avoid friction and deceleration during this phase, the bar should remain as close as possible to the body without touching the thighs until arriving at the peak power position. Once the bar reaches midthigh level, the momentum created in the ascent should be exploited as an explosive triple extension movement as soon as reaching the peak power position reflected by aggressive extension of the hips, knees and ankles ("big jump") while shrugging the shoulders ("bring your shoulders to your ears") (Figure 3a). As result of this aggressive full extension, the athlete should carry forward the momentum of the bar by bringing the elbows up and out (upright row) as the bar continues to
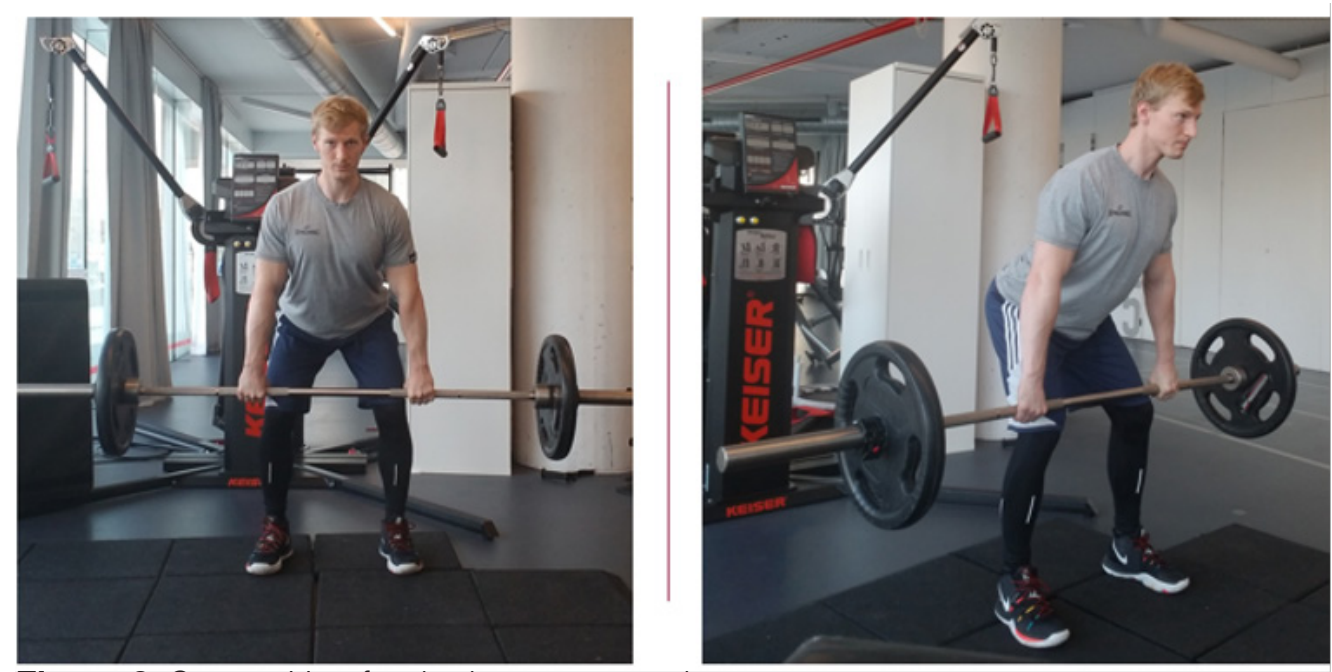

Figure 2. Set position for the hang power clean.
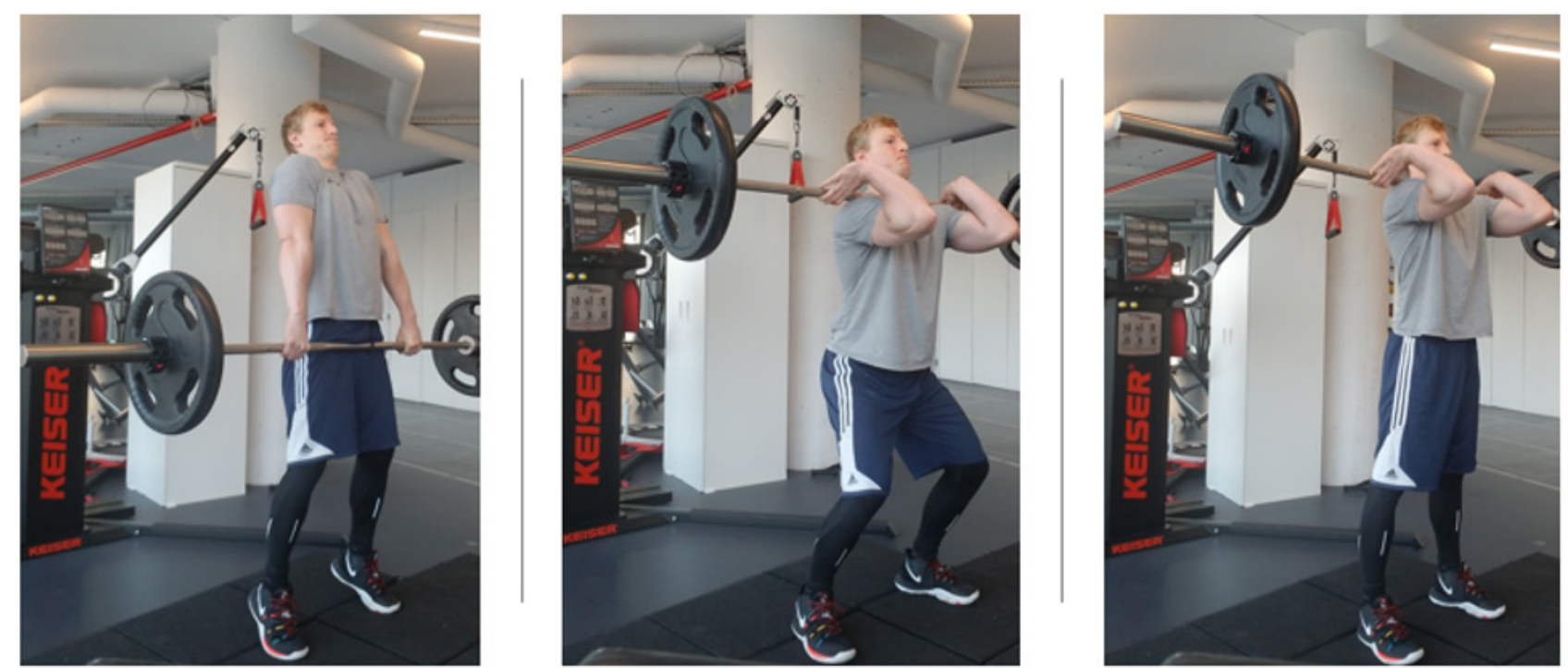

Figure 3. (a) Explosive triple extension movement reflected by aggressive extension of the hips, knees and ankles ("big jump") while shrugging the shoulders. (b) The 'catch' in a front rack position. (c) The 'finish' position. Elbows pointed up and forward into the front squat position. 
move up vertically and close to the body. Finally, the athlete drops his or her body underneath the bar while rapidly shifting the elbows up, out, and around the bar ("rotate your elbows around the bar") [11]. Importantly, the athlete should hold the bar in a relaxed manner to allow greater flexibility in the wrists. This motion allows the athlete to 'catch' the bar in a front rack position landing flat footed (Figure 3b) and finish the entire exercise by driving through the heels with the elbows pointed up and forward into the hang clean and front squat end position (Figure 3c).

3. Common mistakes: Addressing common mistakes and reinforcing proper exercise technique throughout the training year is critical in ensuring player safety, minimizing injury risk, and evoking the appropriate transfer of training stimulus produced by the hang clean $[13,20$, 33]. One of the most common mistakes seen in athletes is pulling with the lower back, because of the shoulders not vertically aligned over the midfoot. To correct this issue, the strength and conditioning coach may instruct the athlete to lean against a robust vertical structure (e.g., squat rack) with the arms hanging forward as a simulation of holding the bar and commencing a hang clean followed by an RDL movement keeping the shoulders in contact with the squat rack (Figure 4). This corrective exercise inherently teaches the athlete to bring the hips forward while keeping the shoulders over the midfoot line [32]. Another common mistake seen in athletes occurs during the final phase of the hang clean as the bar is forcefully received in the catch position. This is often the result of poor movement syntonisation. Therefore, proper timing (receiving the bar at the same moment the bar transitions from ascension to descension) in combination with flexed knees is essential to absorb the load of the bar and avoid unnecessary stress on the body. Additional common errors in technique include: lack of postural integrity (e.g., rounded back); executing the triple extension too early (before the bar reaches midthigh level) causing the bar to fade away from the body or overarching the lower back; 'dipping' under the bar too early (not taking full advantage of the triple extension); pointing the elbows downwards during the 'catch' causing the athlete to lean forward and lose control over the bar [32].

4. Teaching progressions: Traditionally, the 6-step progression model has been suggested as a method to teaching the hang power clean and consists of breaking down the movement (decomposition) in subsequent steps (wholepart-whole method) [11]. However, a 'constraintsled approach' (athlete-centered) to teaching complex movements (exploring complete movements in its entireness) has recently been favoured over the traditional top-down, coach-controlled approaches based upon skill acquisition theory leading to more autonomy by the athlete [36]. Through this constraints-led approach, ecological validity is optimized by adjusting the conditions to perform and refine the lifters hang clean technique, rather than verbally instructing decomposed, isolated, and inherently different skills. Practically, this approach requires
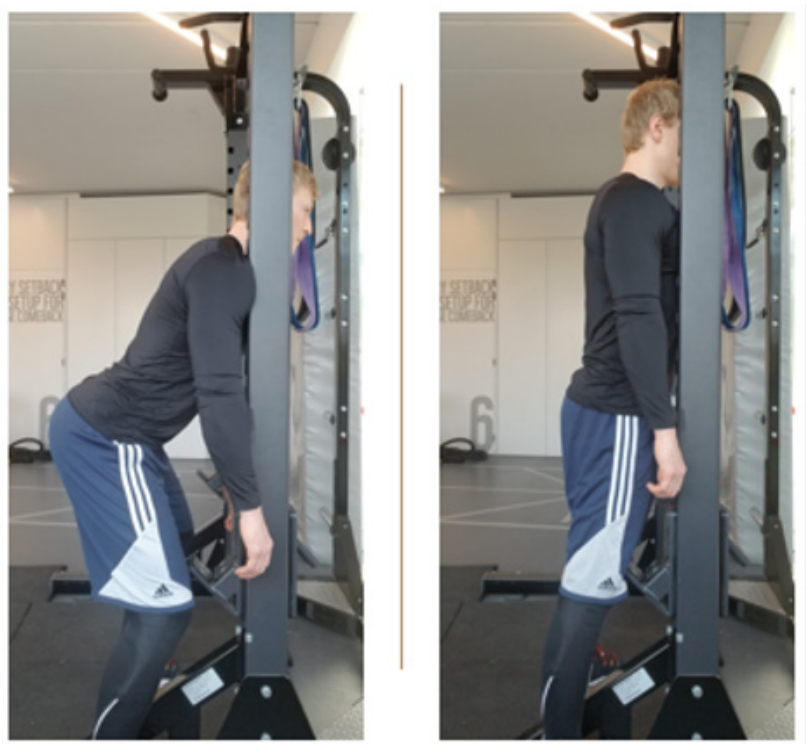

Figure 4. Squat rack corrective exercise. Teaches the athlete to bring the hips forward while keeping the shoulders over the midfoot line. 


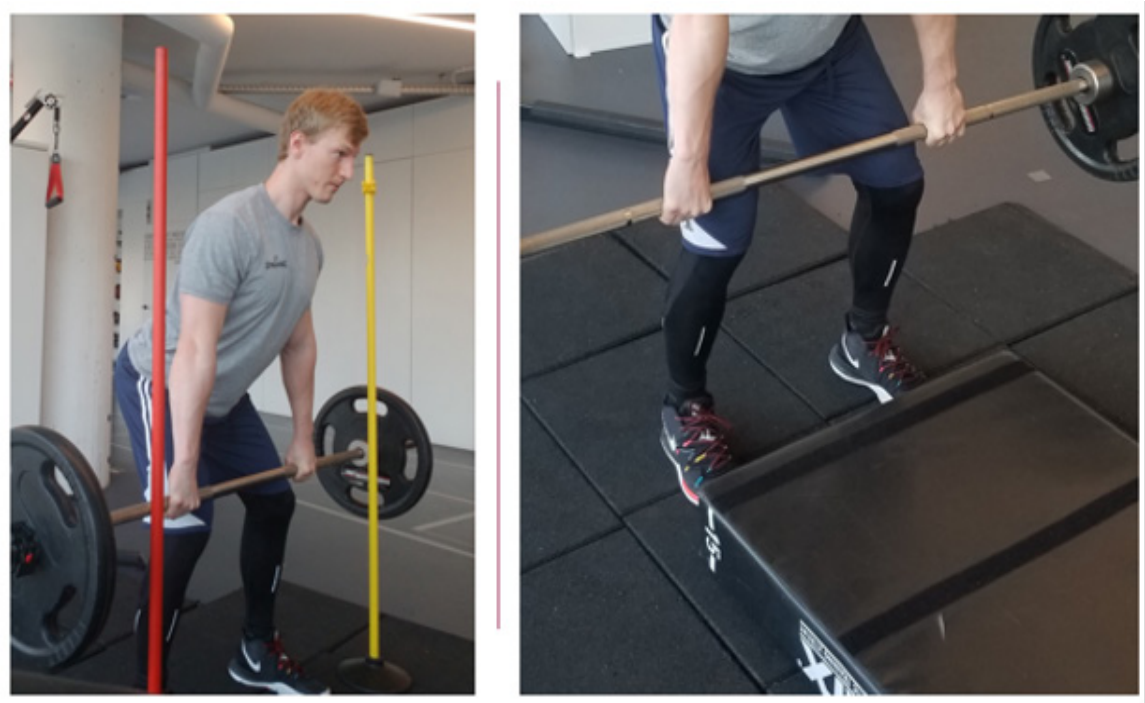

Figure 5. External objects may be used as visual cues to reduce forward barbell passage and excessive horizontal displacement.

coaches to set out problems rather than provide solutions, and design specific conditions for practice from which movement solutions emerge in each athlete respectively [36]. For instance, chalk on the barbell may be applied to show the athlete where contact was made on the thigh and allow exploration of different timings from the start of the second pull. Figure 5 displays external objects (pole rails progressing to a $15-\mathrm{cm}$ mat) in front of the athlete which may be used as visual cues to reduce forward barbell passage and excessive horizontal displacement [36].

\section{Variations}

As with all exercises, there are several variations that can be applied to the power clean. We have used derivatives of the power clean as teaching progressions, while still training the performance qualities in less technically proficient athletes. Examples of power clean variations, derivatives and implements include:

1. Start position. In the sport of weightlifting and in most strength and conditioning programs, the power clean is taught by starting from the floor. However, the start position can be modified based on individual goals, capacities, and environment. The most common start positions are starting at the floor, below the knee, just above the knee and below the hip fold [10, 13, 31]. The start positions may also be taught from a static position or dynamic position [30, 31]. A static position starts from either the floor, blocks, safety bars or a stationary hang position. A dynamic start allows for a countermovement to take place, thus the athlete utilizes the stretch shortening cycle and already has developed a given amount of force before [30].

2. End Position. The typical end position of the power clean is catching the barbell in a front rack position, this is often referred to as the catch phase. The catch phase provides an additional benefit by developing force absorption qualities [31]. However, some athletes report significant wrist discomfort while attempting to catch a loaded barbell in front rack position. The mechanical demands on the wrist and shoulders during the catch phase is a significant consideration in relation to potential risk of injury. According to Suchomel et al., [34] weightlifting derivatives may possess a unique load absorption profile. Research examining the jump shrug and high pull variation demonstrates that eliminating the catch phase may produce comparable or greater force velocity characteristics during the concentric phase of the power clean [22, 28, 32]. Such variations and derivatives may be used, dependent on the technical ability of the athlete and desired strength characteristic targeted.

3. Implement training. There are several implements which can be used to provide clean variations to aid in learning clean technique. These include performing clean variations with kettlebells, dumbbells, sandbags and waterbags (Figure 6), which can be used to complement or augment traditional training [25, 26]. Trap bars or hex bars have also been used to teach clean pull derivatives. The design of the trap bar puts athletes in a much more anatomical advantageous start position by reducing the 

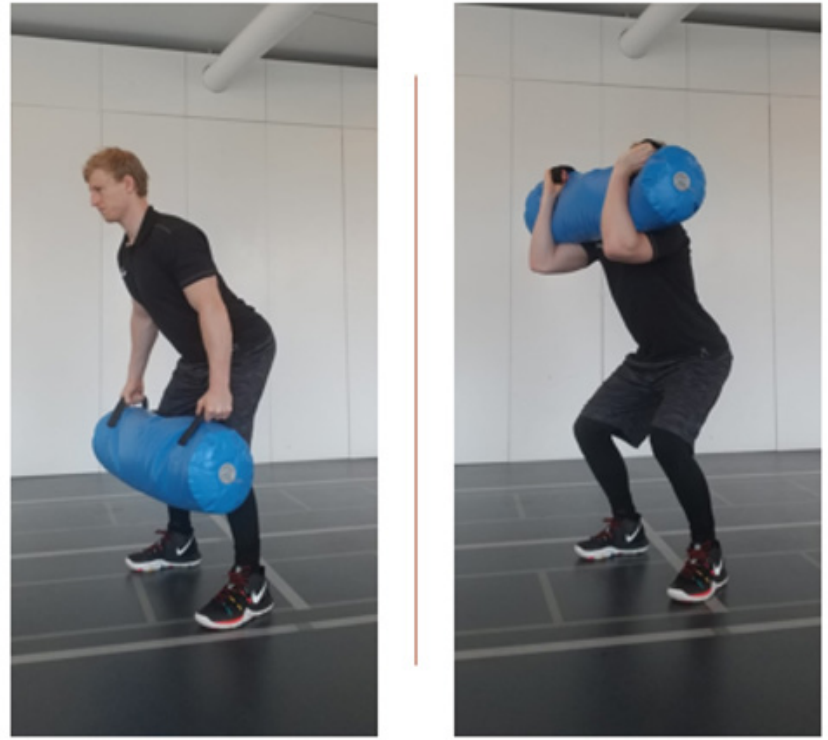

Figure 6. Sandbag hang clean variation.

stress on the lower back, allowing for a more upright set position, which may promote more optimal execution of triple extension in a loaded squat jump [24]. While unstable variations such as sandbags or waterbags, offer perturbative forces that require continuous body stabilization, especially at high velocities. Calatayud et al., [7] reported greater muscle activation of the core when during the waterbag clean in comparison to the traditional barbell version.

\section{CONCLUSION}

Due to its ability to develop total body strength and potential for enhancing athletic performance [19] the hang power clean and power clean are fundamental weightlifting exercises and part of the 'big three' prescribed by strength and conditioning coaches. This includes the squat, deadlift and power clean. The highlighted hang power clean and power clean variations represent advanced, functionally integrated sport-specific applications. For athletes to perform these variations successfully they require significant core strength, proficient deadlift and front squat technique, and unilateral balance. As with the deadlift and squat, the hang power clean and power clean variations are dependent not only on the athletes short and long-term goals, but importantly, their technical lifting competence. For athletes targeting speed strength qualities (i.e., increase power develop), the hang power clean and power clean are an essential component of the training program. Upon mastering the deadlift [3] and front squat [4], an athlete's ability to develop the correct body positioning required in the 4-step teaching progression for the power clean [12] is greatly enhanced. This is often the limiting factor resulting in failure in obtaining the correct catch position of the power clean. It is essential that strength and conditioning coaches prescribing the hang clean, and power clean variations allow time for the athlete to gain technical lifting competence. Through mastery of both the deadlift [3] and front squat [4], the athlete maximizes their potential to gain technical proficiency in the hang power clean and power clean, thereby transferring their athletic abilities from the training floor to the sporting domain.

\section{REFERENCES}

1. Armstrong, D.F. Power training: The key to athletic success. Strength Cond J. 15: 7-11. 1993.

2. Baker, D. and S. Nance The relation between running speed and measures of strength and power in professional rugby league players. J Strength Cond Res. 13: 230-235. 1999.

3. Bird, S.P. and B. Barrington-Higgs Exploring the deadlift. Strength Cond J. 32: 46-51. 2010.

4. Bird, S.P. and S. Casey Exploring the front squat. Strength Cond J. 34: 27-33. 2012.

5. Burdett, R.G. Biomechanics of the snatch technique of highly skilled and skilled weightlifters. Res Q Exerc Sport. 53: 193-197. 1982.

6. Burgener, M. The Power Clean. NSCA Journal. 10: 50-55. 1988.

7. Calatayud, J., J.C. Colado, F. Martin, J. Casaña, M.D. Jakobsen, and L.L. Andersen Core muscle activity during the clean and jerk lift with barbell versus sandbags and water bags. Int J Sports Phys Ther. 10: 803-810. 2015.

8. Comfort, P., M. Allen, and P. Graham-Smith Kinetic comparisons during variations of the power clean. J Strength Cond Res. 25: 3269-3273. 2011.

9. Deweese, B.H., A.J. Serrano, S.K. Scruggs, and 
M.L. Sams The clean pull and snatch pull: Proper technique for weightlifting movement derivatives. Strength Cond J. 34: 82-86. 2012.

10. Deweese, B.H., T.J. Suchomel, A.J. Serrano, J.D. Burton, S.K. Scruggs, and C.B. Taber Pull from the knee: Proper technique and application. Strength Cond J. 38: 79-85. 2016.

11. Duba, J., W.J. Kraemer, and G. Martin A 6-Step progression model for teaching the hang power clean. Strength Cond J. 29: 26-35. 2007.

12. Duba, J., W.J. Kraemer, and G. Martin Progressing from the hang power clean to the power clean: a 4-step model. Strength Cond J. 31: 58-66. 2009.

13. Ebel, K. and R. Rizor Teaching the hang clean and overcoming common obstacles. Strength Cond J. 24: 32-36. 2002.

14. Garhammer, J. Power Clean: Kinesiological Evaluation. NSCA Journal. June-July: 25-28. 1984

15. Gourgoulis, V., N. Aggeloussis, A. Garas, and G. Mavromatis Unsuccessful vs. successful performance in snatch lifts: a kinematic approach. J Strength Cond Res. 23: 486-494 2009.

16. Haff, G.G. and M.H. Stone Methods of developing power with special reference to football players. Strength Cond J. 37: 2-16. 2015.

17. Hedrick, A. Teaching the clean. Strength Cond J. 26 : 70-72. 2004.

18. Hedrick, A. and $\mathrm{H}$. Wada Weightlifting movements: do the benefits outweigh the risks? Strength Cond J. 30: 26-34. 2008.

19. Hori, N., R.U. Newton, W.A. Andrews, N. Kawamori, M.R. Mcguigan, and K. Nosaka Does performance of hang power clean differentiate performance of jumping, sprinting, and changing of direction? J Strength Cond Res. 22: 412-8. 2008.

20. Johnson, J. Teaching the power clean and the hang power clean. NSCA Journal. 4: 52-54. 1982.

21. Kawamori, N., A.J. Crum, P.A. Blumert, J.R. Kulik, J.T. Childers, J.A. Wood, M.H. Stone, and G.G. Haff Influence of different relative intensities on power output during the hang power clean: identification of the optimal load. J Strength Cond Res. 19: 698-708. 2005.

22. Kipp, K., P.J. Malloy, J.C. Smith, M.D. Giordanelli, M.T. Kiely, C.F. Geiser, and T.J. Suchomel Mechanical demands of the hang power clean and jump shrug: A joint-level perspective. J Strength Cond Res. 32: 466-474. 2018.

23. Kipp, K., J. Redden, M.B. Sabick, and C. Harris Weightlifting performance is related to kinematic and kinetic patterns of the hip and knee joints. J Strength Cond Res. 26: 1838-1844. 2012.

24. Lockie, R.G. and A. Lazar Exercise technique: Applying the hexagonal bar to strength and power training. Strength Cond J. 39: 24-32. 2017.

25. Lyons, B.C., J.J. Mayo, W.S. Tucker, B. Wax, and R.C. Hendrix Electromyographical comparison of muscle activation patterns across three commonly performed kettlebell exercises. J Strength Cond Res. 31: 23632370. 2017
26. Manocchia, P., D.K. Spierer, A.K. Lufkin, J. Minichiello, and J. Castro Transference of kettlebell training to strength, power, and endurance. J Strength Cond Res. 27: 477-84. 2013.

27. Newton, H. Power clean: Teaching the beginner Practical applications. NSCA Journal. 6: 37-59. 1984.

28. Oranchuk, D.J., T.L. Robinson, Z.J. Switaj, and E.J. Drinkwater Comparison of the hang high pull and loaded jump squat for the development of vertical jump and isometric force-time characteristics. J Strength Cond Res. 33: 17-24. 2019.

29. Stone, M.H., H.S. O'bryant, F.E. Williams, R.L. Johnson, and K.C. Pierce Analysis of bar paths during the snatch in elite male weightlifters. Strength Cond J. 20: 30-38. 1998.

30. Suchomel, T.J., P. Comfort, and J.P. Lake Enhancing the force-velocity profile of athletes using weightlifting derivatives. Strength Cond J. 39: 10-20. 2017.

31. Suchomel, T.J., P. Comfort, and M.H. Stone Weightlifting pulling derivatives: rationale for implementation and application. Sports Medicne. 45: 823-39. 2015

32. Suchomel, T.J., B.H. Deweese, G.K. Beckham, A.J. Serrano, and S.M. French The hang high pull: A progressive exercise into weightlifting derivatives. Strength Cond J. 36: 79-83. 2014.

33. Suchomel, T.J., B.H. Deweese, and A.J. Serrano The power clean and power snatch from the knee. Strength Cond J. 38: 98-105. 2016.

34. Suchomel, T.J., M.D. Giordanelli, C.F. Geiser, and K. Kipp Comparison of joint work during load absorption between weightlifting derivatives. J Strength Cond Res. 35: S127-S135. 2021.

35. Tufano, J.J., L.E. Brown, and G.G. Haff Theoretical and practical aspects of different cluster set structures: A systematic review. J Strength Cond Res. 31: 848-867. 2017.

36. Verhoeff, W.J., S.K. Millar, A.R.H. Oldham, and J. Cronin Coaching the power clean: A constraints-led approach. Strength Cond J. 42: 16-25. 2020

37. Waller, M., T. Piper, and J. Miller Coaching of the snatch/clean pulls with the high pull variation. Strength Cond J. 31: 47-54. 2009.

38. Whaley, O. and R. Mcclure Another perspective on teaching the pulling movements. Strength Cond J. 19: 58-63. 1997.

39. Winchester, J.B., T.M. Erickson, J.B. Blaak, and J.M. Mcbride Changes in bar-path kinematics and kinetics after power-clean training. J Strength Cond Res. 19: 177-83. 2005

40. Winchester, J.B., J.M. Porter, and J.M. Mcbride Changes in bar path kinematics and kinetics through use of summary feedback in power snatch training. J Strength Cond Res. 23: 444-454. 2009. 